\title{
Synchronization in Networks of Identical Nonlinear Systems via Dynamic Dead Zones
}

\author{
Giacomo Casadei, Daniele Astolfi, Angelo Alessandri, Luca Zaccarian
}

\begin{abstract}
In this paper, we consider the problem of synchronization of a network of nonlinear systems with high-frequency noise affecting the exchange of information. We modify the classic (linear) diffusive coupling by adding dynamic dead zones with the aim of reducing the impact of the noise. We show that the proposed redesign preserves asymptotic synchronization if the noise is not active and we establish a desired ISS property. Simulation results show that, in the presence of noise, the dynamic dead zones highly improve the rejection properties.
\end{abstract}

Index Terms-Synchronization, networks of nonlinear systems, measurement noise, dynamic dead zones.

\section{INTRODUCTION}

C Onsensus and synchronization attracted much attention of the control community since agreement appears as one of the main features in multi-agent systems (and networks) of different nature. One of the first fields of application is the control of multi-agent robotic systems, where the agents have to define a common trajectory to coordinate their motion [1]. At the same time, in many other domains, such as power networks [2] and social networks [3], consensus and synchronization are a natural feature of their dynamics. The problem of linear consensus and synchronization has been widely studied. Major references can be found both on consensus [4] and synchronization of identical linear systems [5]. In recent years, several authors have considered different aspects of the linear framework such as heterogeneous dynamics [6], switching networks [7], and high-order systems [8]. At the same time, the community extended the linear resulst to the nonlinear context. Among others, it is worth mentioning [9], where the author exploits passivity tools, [10] with dissipativity theory, and [11], where the authors use high-gain arguments. Nowadays, many the results of the linear framework have been successfully extended to the nonlinear setting [12], [13].

Synchronization has been redefined often as a stabilization problem of a set describing the desired agreement, for instance by defining a synchronization error variable and

G. Casadei is with Laboratoire Ampere Dpt. EEA of the École Centrale de Lyon, Université de Lyon, 69134 Ecully, France. D. Astolfi is with Université Claude Bernard Lyon 1, CNRS, LAGEPP UMR 5007, 43 boulevard du 11 novembre 1918, F-69100, Villeurbanne, France. A. Alessandri is with University of Genova (DIME), Italy. L. Zaccarian is with Université de Toulouse, CNRS, Toulouse, France, and with Dept. of Industrial Engineering, University of Trento, Trento, Italy.

The research leading to these results is partially funded by ANR via project HANDY, number ANR-18-CE40-0010. studying the stability of its dynamics with respect to the origin. Attention has been given to the analysis of robust stability of the aforementioned set by considering the impact of noise [14], perturbations [15], and hybrid phenomena (such as switching topologies [16] and open networks [17]). However, the definition of control architectures that allow mitigating the effect of these perturbations/phenomena over the networks is a topic widely open. Some authors have exploited $H_{\infty}$ techniques in the case of networks of linear systems [18], [19], while in [20] the authors studied the problem of synchronization exploiting integral quadratic constraints (IQC). However, it is a well known fact that the extension of these techniques to the nonlinear framework presents several obstructions. The problem of high-frequency noise has been considered also in [21] in the context of quantized information, where the authors proposed a hysteretic quantizer.

In recent years, many authors have revisited the design of linear and nonlinear observers by adding nonlinear dynamic filters on the output injection term to reduce the effect of perturbations, such as noise and outliers, affecting the measurement. A notable example of this approach is [22], where the authors studied the use of dynamic saturations to improve the performance of the observer in the presence of impulsive perturbations of the measurement. In the same spirit, in [23] the authors studied the use of dynamic dead zones to deal with high-frequency measurement noise. More recently, the case of nonlinear systems has been considered [24], [25] and simulations show that dead-zone observers can significantly improve performances.

Motivated by these results, in a recently submitted work [26], the authors have considered the use of dynamic saturations to mitigate the impact of impulsive perturbations affecting the communication between linear agents in a network. In this paper, we consider the problem of synchronization in networks of nonlinear systems in presence of high-frequency noise over the communication between agents. We modify the off-the-shelf diffusive coupling by adding dynamic dead zones to reduce the impact of high-frequency noise affecting the communication among the agents. This paper represents a first attempt in studying the benefits of designing nonlinear dynamic couplings in the context of networks of nonlinear systems and displays promising results in reducing the effects of noise affecting the communication.

The paper is structured as follows. In Section II we introduce the synchronization problem at hand and we briefly review the existing results in literature. In Section III, we 
present the dead zone redesign and state the main result of this paper. In Section IV, simulation results are presented.

Notation: $\mathbb{R}$ denotes the set of real numbers and $\mathbb{R}_{>0}=$ $(0, \infty) ;\|\cdot\|$ denotes the standard Euclidean norm; the distance of a vector $x \in \mathbb{R}^{n}$ from a closed set $X \subset$ $\mathbb{R}^{n}$ is denoted as $\|x\|_{X}=\min _{w \in X}\|x-w\| ; \otimes$ denotes the Kronecker product; diag denotes the diagonal operator; col denotes the column operator, namely, given $a_{i} \in \mathbb{R}$, $i=1, \ldots, n$, we have $\operatorname{col}\left(a_{1}, \ldots, a_{n}\right) \in \mathbb{R}^{n \times 1}$. Moreover, given some row vectors $a_{i} \in \mathbb{R}^{1 \times r}, i=1, \ldots, n$, we have $\operatorname{col}\left(a_{1}, \ldots, a_{n}\right) \in \mathbb{R}^{n \times r}$. On $\mathbb{R}^{n}$, we define the row-vector basis $\mathrm{b}_{1}=(1,0, \ldots, 0), \mathrm{b}_{i}=(0, \ldots, 0,1,0, \ldots, 0)$ with all zeros except for a 1 in the $i$-th position, $\mathrm{b}_{n}=(0, \ldots, 0,1)$. Thus, the identity matrix of dimension $n$, denoted by $I_{n}$, is given by $I_{n}=\operatorname{col}\left(\mathrm{b}_{1}, \ldots, \mathrm{b}_{n}\right)$. $\mathbb{1}_{n}$ denotes a column vector of dimension $n$ where all its entries are 1 . Given a matrix $A \in \mathbb{R}^{n \times n}, \lambda_{m}(A)$, respectively $\lambda_{M}(A)$, denotes its minimum, respectively maximum, eigenvalue.

A triplet of matrices $(S, B, C)_{m}$, or simply $(S, B, C)$, is said to be in prime form, if is $S$ is a shift matrix (all 1's on the upper diagonal and all 0's elsewhere) of dimension $m \times m$, $B=\mathrm{b}_{m}^{\top}$ and $C=\mathrm{b}_{1}$. For any $a \in \mathbb{R}, b \in \mathbb{R}_{>0}$, we define the saturation function $\operatorname{sat}_{b}(a):=\min \{b, \max \{\{a,-b\}\}$ and the dead zone function as $\operatorname{dz}_{b}(a):=a-\operatorname{sat}_{b}(a)$. We use the standard notation of input-to-state stability (ISS) and class- $\mathcal{K}$ and class- $\mathcal{K} \mathcal{L}$ functions introduced in [27].

Graph theory: In a general framework, a communication graph is described by a triplet $\mathcal{G}=\{\mathcal{V}, \mathcal{E}, A\}$ in which $\mathcal{V}$ is a set of $n$ nodes $\mathcal{V}=\left\{v_{1}, v_{2}, \ldots, v_{n}\right\}, \mathcal{E} \subset \mathcal{V} \times \mathcal{V}$ is a set of edges $e_{j k}$ that models the interconnection between two nodes with the flow of information from node $j$ to node $k$ weighted by the $(k, j)$-th entry $a_{k j} \geq 0$ of the adjacency matrix $A \in$ $\mathbb{R}^{n \times n}$. We denote by $L \in \mathbb{R}^{n \times n}$ the Laplacian matrix of the graph, with its elements defined as $\ell_{k j}=-a_{k j}$ for $k \neq j$, $\ell_{k j}=\sum_{i=1}^{n} a_{k i}$ for $k=j$. A time-invariant graph is said to be connected if and only if $L$ has only one trivial eigenvalue $\lambda_{1}(L)=0$ and all other eigenvalues $\lambda_{2}(L), \ldots, \lambda_{n}(L)$ have strictly positive real parts (see [28]). Thus with $\mu_{L} \in \mathbb{R}_{>0}$ we denote the positive scalar such that

$$
\operatorname{Re} \lambda_{i}(L) \geq \mu_{L}, \quad \forall i=2, \ldots, n .
$$

Furthermore, we assume the following.

Assumption 1 Given graph $\mathcal{G}$, its Laplacian $L$ is diagonalizable.

We define the following transformation $T \in \mathbb{R}^{n \times n}$ as

$$
T:=\left(\begin{array}{cc}
1 & 0_{1 \times(n-1)} \\
\mathbb{1}_{n-1} & I_{n-1}
\end{array}\right), T^{-1}=\left(\begin{array}{cc}
1 & 0_{1 \times(n-1)} \\
-\mathbb{1}_{n-1} & I_{n-1}
\end{array}\right),
$$

which (from Assumption 1) satisfies

$$
\tilde{L}=T^{-1} L T=\left(\begin{array}{cc}
0 & L_{12} \\
0_{(n-1) \times 1} & L_{22}
\end{array}\right),
$$

where $\operatorname{eig}\left\{L_{22}\right\}=\left\{\lambda_{2}(L), \ldots, \lambda_{n}(L)\right\}$. Also, we will use the following notations

$$
T^{-1} L=\left(\begin{array}{l}
N_{12} \\
N_{22}
\end{array}\right):=\left(\begin{array}{c}
\mathrm{b}_{1} T^{-1} L \\
\operatorname{col}\left(\mathrm{b}_{2}, \ldots, \mathrm{b}_{n}\right) T^{-1} L
\end{array}\right),
$$

$$
L T=\left(\begin{array}{ll}
0 & M
\end{array}\right):=\left(\begin{array}{cc}
0 & M_{1} \\
\vdots & \vdots \\
0 & M_{n}
\end{array}\right), \quad M \in \mathbb{R}^{n \times(n-1)} .
$$

\section{Synchronization Of Nonlinear Systems}

Consider $n$ identical nonlinear agents

$$
\begin{aligned}
\dot{x}_{j} & =S x_{j}+B \phi\left(x_{j}\right)+u_{j} \\
y_{j} & =C x_{j}+D w_{j},
\end{aligned}
$$

where $x_{j} \in \mathbb{R}^{d}$ is the state of the $j$-th agent, $u_{j} \in \mathbb{R}^{d}$ is the diffusive coupling control input to be defined, $(S, B, C)_{d}$ is a triplet in prime form, $\phi: \mathbb{R}^{d} \rightarrow \mathbb{R}$ is globally Lipschitz and $w_{j} \in \mathbb{R}$ is a noise acting on the output $y_{j}$ through the gain $D \in \mathbb{R}$. The agents (6) are connected according to a directed graph $\mathcal{G}=\{\mathcal{V}, \mathcal{E}, A\}$, fulfilling Assumption 1. Moreover, each agent of the network satisfies the following assumption.

Assumption 2 There exists a compact set $X \subset \mathbb{R}^{d}$ such that system (6) is input-to-state stable (ISS) with respect to $u_{j}$ relative to $X$, namely there exist a class- $\mathcal{K} \mathcal{L}$ function $\beta$ and a class- $\mathcal{K}$ function $\gamma$ such that

$$
\left\|x_{j}(t)\right\|_{X} \leq \max \left\{\beta\left(\left\|x_{j}(0)\right\|_{X}, t\right), \gamma\left(\sup _{\tau \in[0, t)}\left\|u_{j}(\tau)\right\|\right)\right\} .
$$

Remark 1 The structure of (6) and the assumption on $\phi(\cdot)$ hold for any uniformly (globally) observable nonlinear system, as discussed in [16, Section 2.2]. It would also be possible to allow functions $\phi$ to be only locally Lipschitz by assuming that all $x_{j}$ evolve in some given compact sets, see also [13, Remark 1].

Under Assumptions 1 and 2, the problem of synchronization, namely of asymptotic convergence to zero of $x_{i}-x_{k}$, for all $i, k \in\{1, \ldots, n\}$, can be addressed in the absence of measurement noise $w_{j}$, via a distributed diffusing coupling design properly assigning the input of the all the agents $j=1, \ldots, n$. Among the different frameworks and designs proposed in literature, see for instance [9]-[10], in this work, we refer in particular to [13]. Therein, it is shown that the synchronization problem is solved by selecting

$$
u_{j}=-G K \sum_{i=1}^{n} \ell_{j i} y_{i}, \quad G=\operatorname{diag}\left(g, g^{2}, \ldots, g^{d}\right),
$$

for suitable selections of $K$ (characterized next in Theorem 1) and $g>0$ and where $\ell_{j i}$ denotes the $(j, i)$ entry of the Laplacian matrix $L$. To suitably characterize the collective behavior, and as a guideline for selecting $K$, it is customary to write in compact form the network interconnection of (6) via (7) by defining

$$
\mathbf{x}:=\operatorname{col}\left(x_{1}, \ldots, x_{n}\right), \quad \mathbf{w}:=\operatorname{col}\left(w_{1}, \ldots, w_{n}\right) .
$$

With this notation, the $\mathrm{x}$ dynamics read

$$
\begin{aligned}
\dot{\mathbf{x}}=\left[\left(I_{n} \otimes S\right)-(L \otimes G K C)\right] \mathbf{x} & +\left(I_{n} \otimes B\right) \Phi(\mathbf{x}) \\
& +(L \otimes G K D) \mathbf{w},
\end{aligned}
$$

where $\Phi(\mathbf{x}):=\operatorname{col}\left(\phi\left(x_{1}\right), \ldots, \phi\left(x_{n}\right)\right)$. Following [13], and by recalling the structure of the matrix $T$ introduced in (2), 
the compact form (9) can be conveniently manipulated by considering the change of variables

$$
\begin{aligned}
\tilde{\mathbf{x}}:=\left(T^{-1} \otimes I_{d}\right) \mathbf{x} & =\left(x_{1}, \mathbf{e}\right) \\
\mathbf{e}:=\left(e_{2}, e_{3}, \ldots, e_{n}\right) & =\left(x_{2}-x_{1}, \ldots, x_{n}-x_{1}\right) .
\end{aligned}
$$

In the $\left(x_{1}, \mathbf{e}\right)$ coordinates, system (9) exhibits a pseudotriangular structure of the form

$$
\begin{aligned}
\dot{x}_{1} & =S x_{1}+B \phi\left(x_{1}\right)-\left(L_{12} \otimes G K C\right) \mathbf{e}+\left(N_{12} \otimes G K D\right) \mathbf{w} \\
\dot{\mathbf{e}} & =A_{g} \mathbf{e}+\Delta \Phi\left(x_{1}, \mathbf{e}\right)+\Gamma_{g} \mathbf{w}
\end{aligned}
$$

where we have used (3), (4) and the following definitions

$$
\begin{aligned}
A_{g} & :=\left(I_{n-1} \otimes S\right)-\left(L_{22} \otimes G K C\right), \\
\Gamma_{g} & :=\left(N_{22} \otimes G K D\right), \\
\Delta \Phi\left(x_{1}, \mathbf{e}\right) & :=\left(I_{n-1} \otimes B\right)\left(\begin{array}{c}
\phi\left(x_{1}+e_{2}\right)-\phi\left(x_{1}\right) \\
\vdots \\
\phi\left(x_{1}+e_{n}\right)-\phi\left(x_{1}\right)
\end{array}\right),
\end{aligned}
$$

thus highlighting the pseudo-triangularity of (11) thanks to the global Lipschitz properties of $\Phi(\cdot)$. It is readily seen that in absence of measurement noise $\mathbf{w}$, assessing global exponential stability of e (namely exponential convergence to zero of the error coordinates) guarantees asymptotic synchronization of the agents to the perturbed trajectory of $x_{1}$ which is bounded due to Assumption 2. Thus convergence to the forward invariant set $X$ is established. To this end, we recall the result from [13], which provides a suitable choice of the diffusing coupling $K$ establishing exponential stability of the e-dynamics (11). We also report the main steps of the proof given in [13] which are instrumental to the main result of this work, see Section III. Furthermore, we show that the resulting network dynamics is also input-to-state stable (ISS) with respect to $\mathbf{w}$.

Theorem 1 Suppose Assumptions 1 and 2 hold. Let $K$ in (7) be selected as ${ }^{1}$

$$
K=P C^{\top}, \quad P S^{\top}+S P-2 \mu_{L} P C^{\top} C P+a I=0,
$$

with $\mu_{L}$ defined in (1) and for some $a>0$. Then, there exists $g^{\star} \geq 1$ such that, for any $g>g^{\star}$, the network (9) is ISS with respect to $\mathbf{w}$ relative to the compact set

$$
\begin{aligned}
\mathbf{X}:=\left\{\left(x_{1}, x_{2}, \ldots, x_{n}\right) \in X \times \cdots \times X:\right. \\
\left.x_{1}=x_{2}=\cdots=x_{N}\right\} .
\end{aligned}
$$

Proof: The proof is an immediate generalization of the construction in [13]. First of all, let us define the following change of coordinates

$$
\mathbf{e} \mapsto \varepsilon:=\operatorname{col}\left(\varepsilon_{2}, \ldots, \varepsilon_{n}\right):=g\left(I_{n} \otimes G^{-1}\right) \mathbf{e} .
$$

By using the fact that $G^{-1} S G=g S, C G=g C$ and $G^{-1} B=g^{-d} B$, the $\varepsilon$ dynamics read

$$
\dot{\varepsilon}=g A \varepsilon+\Delta \widetilde{\Phi}\left(x_{1}, \varepsilon\right)+g \Gamma \mathbf{w}
$$

\footnotetext{
${ }^{1}$ As shown in [13], the solution to the algebraic Riccati equation always admits a positive definite solution $P$ for any pair of $a, \mu_{L}$.
}

where we defined

$$
\begin{aligned}
A & :=\left(I_{n-1} \otimes S\right)-\left(L_{22} \otimes K C\right), \\
\Gamma & :=\left(N_{22} \otimes K D\right), \\
\Delta \widetilde{\Phi}\left(x_{1}, \varepsilon\right) & =\frac{\left(I_{n-1} \otimes B\right)}{g^{(d-1)}}\left(\begin{array}{c}
\phi\left(x_{1}+g^{-1} G \varepsilon_{2}\right)-\phi\left(x_{1}\right) \\
\vdots \\
\phi\left(x_{1}+g^{-1} G \varepsilon_{n}\right)-\phi\left(x_{1}\right)
\end{array}\right) .
\end{aligned}
$$

Since $\phi$ is globally Lipschitz, there exists $\varrho>0$ such that

$$
\left\|\Delta \widetilde{\Phi}\left(x_{1}, \varepsilon\right)\right\| \leq \varrho\|\varepsilon\| \quad \forall\left(\varepsilon, x_{1}\right) \in \mathbb{R}^{d(n-1)} \times \mathbb{R}^{d} .
$$

Now, following [13, App. A] and thanks to Assumption 1, we know there exists an invertible complex matrix $Q \in \mathbb{C}^{n \times n}$ such that $Q L_{22} Q^{-1}=\operatorname{diag}\left(\lambda_{2}, \ldots, \lambda_{n}\right)$. With the matrix $P=P^{\top}>0$ solution to (13), we define the quadratic Lyapunov function

$$
V(\varepsilon):=\varepsilon^{\top}\left(Q^{*} Q \otimes P^{-1}\right) \varepsilon=\varepsilon^{\top}\left(H_{R} \otimes P^{-1}\right) \varepsilon,
$$

where $Q^{*}$ is the conjugate transpose of $Q$ and $H_{R}:=$ $\operatorname{Re}\left(Q^{*} Q\right)$ is Hermitian and positive definite by construction. From the property of the Kronecker product, we obtain

$$
\underline{\alpha}^{2}\|\varepsilon\|^{2} \leq V(\varepsilon) \leq \bar{\alpha}^{2}\|\varepsilon\|^{2},
$$

with the positive scalars $\underline{\alpha}^{2}:=\lambda_{m}\left(H_{R}\right) \lambda_{M}(P)^{-1}>0$ and $\bar{\alpha}^{2}:=\lambda_{M}\left(H_{R}\right) \lambda_{m}(\bar{P})^{-1}>0$. Moreover, from the calculations in [13, page 2689], there exists $\bar{a}>0$, possibly depending on $P$, such that $P^{-1} F_{i}+F_{i}^{*} P^{-1}<-\bar{a} I$, where $F_{i}:=S-\lambda_{i} K C$. Then, according to [13, eqn. (36)], we get

$$
\begin{gathered}
\dot{V}=2 \varepsilon^{\top}\left(H_{R} \otimes P^{-1}\right)\left(g A \varepsilon+\Delta \widetilde{\Phi}\left(x_{1}, \varepsilon\right)\right) \\
\quad+2 g \varepsilon^{\top}\left(H_{R} \otimes P^{-1}\right) \Gamma \mathbf{w} \\
\leq-g \bar{a} \varepsilon^{\top}\left(H_{R} \otimes I_{d}\right) \varepsilon+2\|\varepsilon\|\left\|H_{R}\right\|\left\|P^{-1}\right\|\left\|\Delta \widetilde{\Phi}\left(x_{1}, \varepsilon\right)\right\| \\
\quad+2 g\|\varepsilon\|\left\|H_{R}\right\|\left\|P^{-1}\right\|\|\Gamma\|\|\mathbf{w}\| \\
\leq-\left(g \bar{a} \underline{\alpha}^{2}-2 \varrho\left\|H_{R}\right\|\left\|P^{-1}\right\|\right)\|\varepsilon\|^{2}+2 g\|\varepsilon\| \bar{\alpha} \gamma\|\mathbf{w}\|
\end{gathered}
$$

where in the last step we have used the (18) and we denoted $\gamma=\|\Gamma\|$. Let $g^{\star}=2 \varrho\left\|H_{R}\right\|\left\|P^{-1}\right\| /(\bar{a} \underline{\alpha})$ and select any $g>g^{\star}$. Then, using $c:=\bar{a} \underline{\alpha}-2 g^{-1} \varrho\left\|H_{R}\right\|\left\|P^{-1}\right\|>0$ the previous inequality can be bounded as

$$
\dot{W} \leq-g c\|\varepsilon\|^{2}+2 g\|\varepsilon\| \bar{\alpha} \gamma\|\mathbf{w}\| .
$$

As already established in [13], when $\mathbf{w}=0$, the triangular structure in (11), Assumption 2 applied to the $x_{1}$-dynamics, and inequality (22) allow concluding global exponential stability of the set $\mathbf{X}$. Moreover, when $\mathbf{w} \neq 0$, ISS of the set $\mathbf{X}$ can be proved by using again (22) and standard Lypaunov arguments (see, e.g., [29, Chapter 10] for further details).

Remark 2 By inspecting the proof of Theorem 1, we see that the choice of the gain $g$ might be critical. A large $g$ allows to dominate the nonlinear contribution to the dynamics, thus enforcing stability of $\varepsilon$. However, since the ISS-gain with input $\mathbf{w}$ is proportional to $g$, see (22), disturbance rejection performances degrade for an increasing $g$. 


\section{Redesign via Dynamic Dead Zones}

In order to improve the behavior in the presence of measurement noise, we follow the idea first proposed in [23], [25], by introducing a dead zone in the communication among the agents. In particular, by a similar design as in Theorem 1, we replace the linear selection in (7) by

$u_{j}=-G K \mathrm{dz}_{\sqrt{\sigma_{j}}}\left(\sum_{i=1}^{n} \ell_{j i} y_{i}\right), \quad G=\operatorname{diag}\left(g, g^{2}, \ldots, g^{d}\right)$,

where the dead zone level $\sigma_{j}$ is driven by the diffusing coupling input $\sum_{i} \ell_{j i} y_{i}$ itself, namely it obeys the dynamics

$$
\dot{\sigma}_{j}=-g \theta \sigma_{j}+g r\left(\sum_{i=1}^{n} \ell_{j i} y_{i}\right)^{2}, \quad \sigma_{j} \in \mathbb{R}_{\geq 0},
$$

in which $\theta, r>0$ are parameters to be properly chosen and $\sigma_{j}(0)$ is constrained to evolve in the forward invariant set $\mathbb{R}_{\geq 0}{ }^{2}$. Intuitively, the dead zone level $\sigma_{j}$ evolves according to the diffusive coupling $\sum_{i} \ell_{j i} y_{i}$ and converges to zero when the diffusive coupling goes to zero, namely when synchronization is achieved.

In order to derive a more compact notation for the closedloop system, we define the following variables

$$
\begin{aligned}
\zeta_{j} & :=\sum_{i=1}^{n} \ell_{j i} y_{i}=\sum_{i=1}^{n} \ell_{j i}\left(C x_{i}+D w_{i}\right) \\
& =\left(\mathrm{b}_{j} L \otimes C\right) \mathbf{x}+\left(\mathrm{b}_{j} L \otimes D\right) \mathbf{w}, j=1, \ldots, n \\
\zeta & :=\operatorname{col}\left(\zeta_{1}, \ldots, \zeta_{n}\right)=(L \otimes C) \mathbf{x}+(L \otimes D) \mathbf{w} .
\end{aligned}
$$

As a consequence, by using the notation (8), the network interconnection of (6) via (23), (24), can be written in the following compact form

$$
\begin{aligned}
& \dot{\mathbf{x}}=\left(I_{n} \otimes S\right) \mathbf{x}+\left(I_{n} \otimes B\right) \Phi(\mathbf{x})-\left(I_{n} \otimes G K\right) \mathbf{d z}_{\sqrt{\boldsymbol{\sigma}}}(\boldsymbol{\zeta}), \\
& \dot{\boldsymbol{\sigma}}=-g \Theta \boldsymbol{\sigma}+g \operatorname{diag}(\boldsymbol{\zeta}) R \boldsymbol{\zeta}, \quad \boldsymbol{\sigma} \in \mathbb{R}_{\geq 0}^{n},
\end{aligned}
$$

where $\boldsymbol{\sigma}:=\left(\sigma_{1}, \ldots, \sigma_{n}\right), \Theta:=\operatorname{diag}(\theta, \ldots, \theta), R:=$ $\operatorname{diag}(r, \ldots, r)$, and $\mathbf{d} \mathbf{z}_{\sqrt{\sigma}}(\cdot)$ is defined as

$$
\mathbf{d z}_{\sqrt{\sigma}}(\zeta):=\operatorname{col}\left(\mathrm{dz}_{\sqrt{\sigma_{1}}}\left(\zeta_{1}\right), \ldots, \mathrm{dz}_{\sqrt{\sigma_{n}}}\left(\zeta_{n}\right)\right) .
$$

In the next theorem, we show that the dead zone design (23), (24) preserves the same properties stated in Theorem 1.

Theorem 2 Consider the network of $n$ agents (6), (23), (24) (equivalently (26)) and suppose Assumptions 1 and 2 hold. Let $K$ and $g$ be chosen according to Theorem 1. Then, for any $r>0$, there exists $\theta^{\star}>0$ such that for any $\theta \geq \theta^{\star}$, the network (26) is ISS with respect to $\mathbf{w}$ relative to the set $\mathbf{X}$ defined in (14).

Proof: First, let us introduce the following identity

$$
\mathrm{dz}_{\sqrt{\sigma}}(\zeta)=\zeta-\operatorname{sat}_{\sqrt{\sigma}}(\zeta)
$$

where $\operatorname{sat}_{\sqrt{\sigma}}(\cdot)$ is defined as

$$
\operatorname{sat}_{\sqrt{\sigma}}(\zeta):=\operatorname{col}\left(\operatorname{sat}_{\sqrt{\sigma_{1}}}\left(\zeta_{1}\right), \ldots, \operatorname{sat}_{\sqrt{\sigma_{n}}}\left(\zeta_{n}\right)\right) .
$$

\footnotetext{
${ }^{2}$ The arising constrained differential equation well fits the Lyapunov Theory in [30, Theorem 3.18].
}

As a consequence, by using the definition of $\zeta$ in (25), we rewrite the $\mathrm{x}$-dynamics in (26) as

$$
\begin{aligned}
& \dot{\mathbf{x}}=\left(I_{n} \otimes S\right) \mathbf{x}+\left(I_{n} \otimes B\right) \Phi(\mathbf{x}) \\
&-\left(I_{n} \otimes G K\right)\left(\boldsymbol{\zeta}-\mathbf{s a t}_{\sqrt{\sigma}}(\boldsymbol{\zeta})\right) \\
&=\left[\left(I_{n} \otimes S\right)-(L \otimes G K C)\right] \mathbf{x}+\left(I_{n} \otimes B\right) \Phi(\mathbf{x}) \\
& \quad-(L \otimes G K D) \mathbf{w}+\left(I_{n} \otimes G K\right) \mathbf{s a t}_{\sqrt{\sigma}}(\boldsymbol{\zeta}),
\end{aligned}
$$

As in Section II, we apply the change of coordinates $\tilde{\mathbf{x}}=$ $\left(T^{-1} \otimes I_{d}\right) \mathbf{x}$ with $T$ as in (2) and we recall the structure (10). Note that, in light of (25), the variables $\zeta_{i}$ are defined in the new coordinates as

$$
\begin{aligned}
\zeta_{i} & =\left(\mathrm{b}_{i} L \otimes C\right) \mathbf{x}+\left(\mathrm{b}_{i} L \otimes D\right) \mathbf{w} \\
& =\mathrm{b}_{i}(L T \otimes C) \tilde{\mathbf{x}}+\left(\mathrm{b}_{i} L \otimes D\right) \mathbf{w} \\
& =\left(M_{i} \otimes C\right) \mathbf{e}+\left(\mathrm{b}_{i} L \otimes D\right) \mathbf{w},
\end{aligned}
$$

for all $i=1, \ldots, n$, in which the matrices $M_{i}$ are defined as in (5). As a consequence, by collecting the $\zeta_{i}$ defined in (28), and by using the definition of $M$ given in (5), we obtain

$$
\boldsymbol{\zeta}=(L T \otimes C) \tilde{\mathbf{x}}+(L \otimes D) \mathbf{w}=(M \otimes C) \mathbf{e}+(L \otimes D) \mathbf{w},
$$

namely the variable $\zeta$ is independent of $x_{1}$. Similarly to (11), the $\tilde{\mathbf{x}}=\left(x_{1}, \mathbf{e}\right)$-dynamics are obtained from (27) and (29) as follows

$$
\begin{aligned}
\dot{x}_{1} & =S x_{1}+B \phi\left(x_{1}\right)-G K \mathrm{dz}_{\sqrt{\sigma_{1}}}\left(\zeta_{1}\right) \\
\dot{\mathbf{e}} & =A_{g} \mathbf{e}+\Delta \Phi\left(x_{1}, \mathbf{e}\right)+K_{\mathbf{e}} \mathbf{s a t}_{\sqrt{\boldsymbol{\sigma}}}(\boldsymbol{\zeta})-\Gamma_{g} \mathbf{w} \\
\dot{\boldsymbol{\sigma}} & =-g \Theta \boldsymbol{\sigma}+g \operatorname{diag}(\boldsymbol{\zeta}) R \boldsymbol{\zeta}, \quad \boldsymbol{\sigma} \in \mathbb{R}_{\geq 0}^{n},
\end{aligned}
$$

with $A_{g}, \Gamma_{g}, \Delta \Phi$ defined in (12), and where we denoted $K_{\mathbf{e}}:=\left(\operatorname{col}\left(\mathrm{b}_{2}, \ldots, \mathrm{b}_{n}\right) T^{-1} \otimes G K\right)$. Finally, as in the proof of Theorem 1, we apply the change of coordinates (15) to the e-dynamics in (30), and we ignore for the time being the $x_{1}$-dynamics. Note that $\left(M \otimes C G^{-1} g\right)=(M \otimes C)$, which implies $\zeta=(M \otimes C) \varepsilon+(L \otimes D) \mathbf{w}$. As a consequence, similarly to (16), by using the definitions of $A, \Gamma, \widetilde{\Phi}$ given in (17), we obtain the following dynamics in the $\varepsilon$-coordinates

$$
\begin{aligned}
\dot{\boldsymbol{\varepsilon}} & =g A \boldsymbol{\varepsilon}+\Delta \widetilde{\Phi}\left(x_{1}, \boldsymbol{\varepsilon}\right)+g K_{\varepsilon} \mathbf{s a t}_{\sqrt{\boldsymbol{\sigma}}}(\boldsymbol{\zeta})-g \Gamma \mathbf{w} \\
\dot{\boldsymbol{\sigma}} & =-g \Theta \boldsymbol{\sigma}+g \operatorname{diag}(\boldsymbol{\zeta}) R \boldsymbol{\zeta}, \quad \boldsymbol{\sigma} \in \mathbb{R}_{\geq 0}^{n},
\end{aligned}
$$

with $K_{\varepsilon}:=\left(\operatorname{col}\left(\mathrm{b}_{2}, \ldots, \mathrm{b}_{n}\right) T^{-1} \otimes K\right)$. Consider now the Lyapunov function, independent of $x_{1}$, defined as $W(\varepsilon, \boldsymbol{\sigma})=$ $V(\varepsilon)+\nu \mathbb{1}_{n}^{\top} \boldsymbol{\sigma}$, with $V$ defined as in (19) and $\nu>0$ a parameter to be defined. Following (21), we compute its time derivative as

$$
\begin{aligned}
\dot{W} \leq & -\left(g \bar{a} \underline{\alpha}-2 \varrho\left\|H_{R}\right\|\left\|P^{-1}\right\|\right)\|\varepsilon\|^{2} \\
& +2 g \varepsilon^{\top}\left(H_{R} \otimes P^{-1}\right)\left(K_{\varepsilon} \operatorname{sat}_{\sqrt{\boldsymbol{\sigma}}}(\boldsymbol{\zeta})-\Gamma \mathbf{w}\right) \\
& -g \nu \mathbb{1}_{n}^{\top} \Theta \boldsymbol{\sigma}+g \nu \boldsymbol{\zeta}^{\top} R \boldsymbol{\zeta} .
\end{aligned}
$$

By using the definition of the saturation function, we have the following property

$$
\left\|\operatorname{sat}_{\sqrt{\boldsymbol{\sigma}}}(\boldsymbol{\zeta})\right\| \leq \sqrt{\sum_{j=1}^{n}\left(\sqrt{\sigma_{j}}\right)^{2}}=\sqrt{\mathbb{1}_{n}^{\top} \boldsymbol{\sigma}} .
$$

Furthermore, by using the definition of $\zeta$ and the Young inequality $^{3}$, there exists $m, \delta>0$ such that $\|\boldsymbol{\zeta}\|^{2} \leq m\|\varepsilon\|^{2}+$

\footnotetext{
${ }^{3}$ The Young inequality is the well-known upper bound $2 a b \leq \nu a^{2}+b^{2} / \nu$, holding for any $a, b \in \mathbb{R}$ and any $\nu>0$.
} 
$\delta\|\mathbf{w}\|^{2}$. Finally let $\beta:=\left\|\left(H_{R} \otimes P^{-1}\right) K_{\varepsilon}\right\|$. Now let $g$ be fixed so that $g>g^{\star}$ with $g^{\star}$ given by Theorem 1. As a consequence, by using previous bounds, inequality (22), and again the Young inequality for the coupling term in $\sigma$ and $\varepsilon$, the time derivative of $W$ can be upper-bounded as follows:

$$
\begin{aligned}
& \dot{W} \leq-g c\|\varepsilon\|^{2}+2 g\|\varepsilon\| \beta \sqrt{\mathbb{1}_{n}^{\top} \boldsymbol{\sigma}}+2 g\|\varepsilon\| \bar{\alpha} \gamma\|\mathbf{w}\| \\
&-g \nu \theta \mathbb{1}_{n}^{\top} \boldsymbol{\sigma}+g \nu r\left(m\|\varepsilon\|^{2}+\delta\|\mathbf{w}\|^{2}\right) \\
& \leq-g(c-\nu-\nu r m)\|\varepsilon\|^{2}-g\left(\nu \theta-\frac{1}{\nu} \beta^{2}\right) \mathbb{1}_{n}^{\top} \boldsymbol{\sigma} \\
&+2 g\|\varepsilon\| \bar{\alpha} \gamma\|\mathbf{w}\|+g \nu r \delta\|\mathbf{w}\|^{2} .
\end{aligned}
$$

Let $r>0$ be fixed and select $\nu=\frac{c}{2}(1+r m)^{-1}>0$ and $\theta \geq \theta^{\star}:=\nu^{-1}\left(\frac{c}{2}+\frac{\beta^{2}}{\nu}\right)$. We obtain

$$
\dot{W} \leq-\frac{g c}{2}\left(\|\varepsilon\|^{2}+\mathbb{1}_{n}^{\top} \boldsymbol{\sigma}\right)+2 g\|\varepsilon\| \bar{\alpha} \gamma\|\mathbf{w}\|+g \nu r \delta\|\mathbf{w}\|^{2} .
$$

When $\mathbf{w}=0$, the last inequality implies that $\varepsilon$ and $\boldsymbol{\sigma}$ converge exponentially to zero by standard Lyapunov arguments. Since the $x_{1}$-component is ISS with respect to $\zeta_{1}$ (see Assumption 2), we conclude that the set $\mathbf{X}$ defined in (14) is globally exponentially stable. When $\mathbf{w} \neq 0$, inequality (33) and the ISS property of Assumption 2 allow to conclude that the closed-loop system is also ISS relative to the set $\mathbf{X}$.

\section{Simulation Result}

We consider here the example proposed in [13]. In particular, we select (6) as a network of $n=5$ Van der Pol oscillators, whose dynamics are given by

$$
\begin{aligned}
\dot{x}_{j_{1}} & =x_{j_{2}}+u_{j_{1}} \\
\dot{x}_{j_{2}} & =-\left(1-x_{j_{1}}^{2}\right) x_{j_{2}}+x_{j_{1}}+u_{j_{2}} \\
y_{j} & =x_{j_{1}}+w_{j}
\end{aligned}
$$

exchanging their output information $y_{j}$ with their neighbors and with control inputs $u_{j}=\left(u_{j_{1}}, u_{j_{2}}\right)^{\top}$ defined according to (7) and $K$ chosen according to Theorem 1 with $g=10$ and $a=2$. The same network is then considered by replacing (7) with the dynamic dead zones designed according to (23)(24), with $\theta=30$ and $r=25$. The network considered in this example is described by the Lapalacian matrix

$$
L=\left(\begin{array}{ccccc}
-2 & 1 & 1 & 0 & 0 \\
1 & -3 & 1 & 0 & 1 \\
0 & 1 & -2 & 0 & 1 \\
0 & 0 & 1 & -2 & 1 \\
0 & 1 & 0 & 0 & -1
\end{array}\right)
$$

A white noise $\left|w_{j}\right| \leq 1$, acting on the communication is considered. At $t=0 \mathrm{sec}$ we add the white noise to the output of one of the oscillators. The noise persists for $10 \mathrm{sec}$ and then vanishes. Sequentially, each oscillator's output is affected. Last, at $t=100 \mathrm{sec}$, the white noise affects all the communication between the agents. Simulation results are shown in Figure 1, both for the classic design and the redesign proposed in Section III.

We can observe that with both designs the network converges to practical synchronization. The results in Figure 1(a) show clearly that, by redesigning the control input with our dynamic dead zone, the impact of the noise is highly reduced both during the transient and in steady-state. This can be observed also by looking at the norm $\left\|u_{j}\right\|$ of the control input in Figure 1(b). Without redesign, the noise has a strong impact on the synchronization input. Such improvement results to be even more visible for larger choices of $g$. By looking at the local values of the threshold $\sigma_{j}$ in Figure 2, it is possible to observe how differently the dynamic dead zones operate depending on which output is affected by the noise. Last, we observe that when all communication channels are affected by the noise, the standard design fails to guarantee synchronization over the nominal Van der Pol trajectory while the redesign preserves such trajectory.

\section{Conclusions}

In this paper, we proposed the use of dynamic dead zones to reduce the effect of high-frequency noise affecting the communication between nonlinear agents in a network. We have shown that a dead zone redesign allows to preserve the synchronization properties while improving the performance of the network. Numerical simulations reveal a concrete potential to reduce the ISS gain from the measurement noise to the synchronization errors, although the quantitative characterization of this ISS gain appears to be a nontrivial task due to the convoluted nonlinear effect of the dynamic dead zone.

This work can be extended in many directions. First, by considering a wider class of systems and nominal diffusive couplings. Second, by considering the use of other dynamic filters, such as dynamic saturations, to take into account more complex perturbations affecting the network. With this respect, we plan to extend the approach presented in [26] to the nonlinear framework.

\section{REFERENCES}

[1] R. Olfati-Saber and R. M. Murray, "Consensus problems in networks of agents with switching topology and time-delays," IEEE Transactions on automatic control, vol. 49, no. 9, pp. 1520-1533, 2004.

[2] F. Dörfler, M. Chertkov, and F. Bullo, "Synchronization in complex oscillator networks and smart grids," Proceedings of the National Academy of Sciences, vol. 110, no. 6, pp. 2005-2010, 2013.

[3] P. Jia, A. Mirtabatabaei, N. E. Friedkin, and F. Bullo, "Opinion dynamics and the evolution of social power in influence networks," SIAM review, vol. 57, no. 3, pp. 367-397, 2015.

[4] L. Moreau, "Stability of continuous-time distributed consensus algorithms," in Decision and Control, 2004. CDC. 43rd IEEE Conference on, vol. 4. IEEE, 2004, pp. 3998-4003.

[5] L. Scardovi and R. Sepulchre, "Synchronization in networks of identical linear systems," in Decision and Control, 2008. CDC 2008. 47th IEEE Conference on. IEEE, 2008, pp. 546-551.

[6] P. Wieland, R. Sepulchre, and F. Allgöwer, "An internal model principle is necessary and sufficient for linear output synchronization," Automatica, vol. 47, no. 5, pp. 1068-1074, 2011.

[7] J. Lu, D. W. Ho, J. Cao, and J. Kurths, "Exponential synchronization of linearly coupled neural networks with impulsive disturbances," IEEE Transactions on Neural Networks, vol. 22, no. 2, pp. 329-336, 2011.

[8] J. H. Seo, H. Shim, and J. Back, "Consensus of high-order linear systems using dynamic output feedback compensator: Low gain approach," Automatica, vol. 45, no. 11, pp. 2659-2664, 2009.

[9] M. Arcak, "Passivity as a design tool for group coordination," IEEE Transactions on Automatic Control, vol. 52, no. 8, pp. 1380-1390, 2007.

[10] G.-B. Stan and R. Sepulchre, "Analysis of interconnected oscillators by dissipativity theory," IEEE Transactions on Automatic Control, vol. 52, no. 2, pp. 256-270, 2007. 

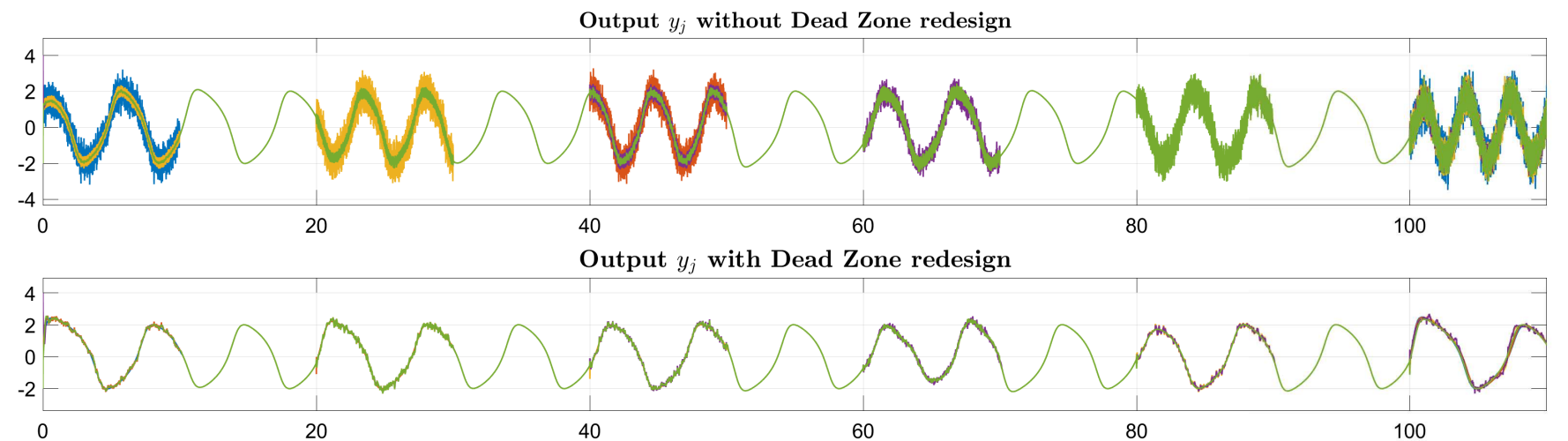

(a)
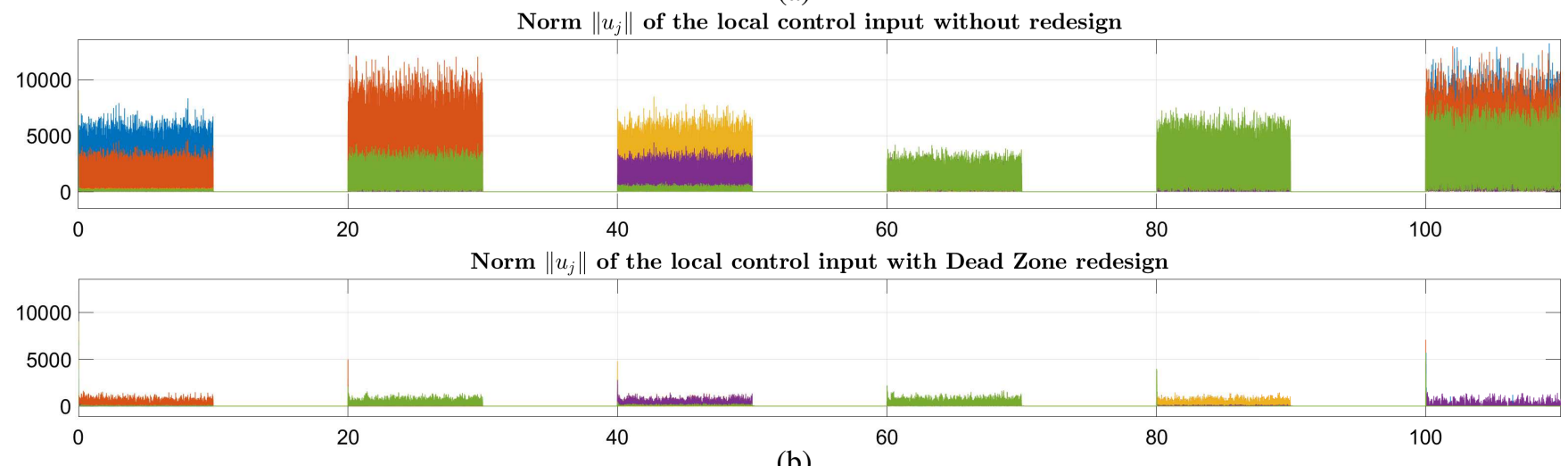

(b)

Fig. 1. In Figure 1(a), a comparison between the synchronization without coupling redesign and with coupling redesign is shown. In Figure 1(b), the norm of the inputs $\left\|u_{j}\right\|$ without redesign and with dead zone redesign is shown.

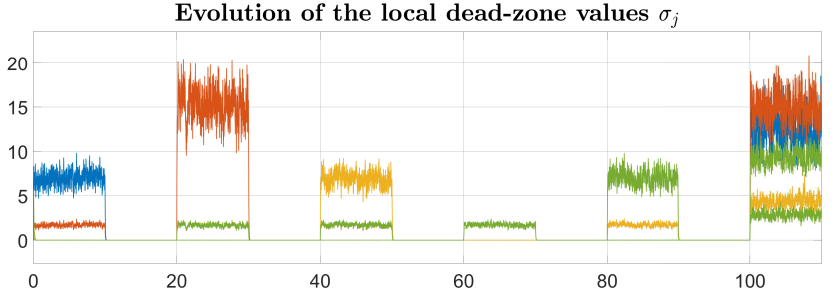

Fig. 2. The evolution of the local dead zone values $\sigma_{j}$.

[11] E. Panteley and A. Loría, "Synchronization and dynamic consensus of heterogeneous networked systems," IEEE Transactions on Automatic Control, vol. 62, no. 8, pp. 3758-3773, 2017.

[12] A. Abdessameud, I. G. Polushin, and A. Tayebi, "Synchronization of nonlinear systems with communication delays and intermittent information exchange," Automatica, vol. 59, pp. 1-8, 2015.

[13] A. Isidori, L. Marconi, and G. Casadei, "Robust output synchronization of a network of heterogeneous nonlinear agents via nonlinear regulation theory," IEEE Transactions on Automatic Control, vol. 59, no. 10, pp. 2680-2691, 2014.

[14] J.-n. Teramae and D. Tanaka, "Robustness of the noise-induced phase synchronization in a general class of limit cycle oscillators," Physical review letters, vol. 93, no. 20, p. 204103, 2004.

[15] J. Zhou, L. Xiang, and Z. Liu, "Synchronization in complex delayed dynamical networks with impulsive effects," Physica A: Statistical Mechanics and Its Applications, vol. 384, no. 2, pp. 684-692, 2007.

[16] G. Casadei, A. Isidori, and L. Marconi, "About disconnected topologies and synchronization of homogeneous nonlinear agents over switching networks," International Journal of Robust and Nonlinear Control, vol. 28, no. 3, pp. 901-917, 2018.

[17] J. M. Hendrickx and S. Martin, "Open multi-agent systems: Gossiping with random arrivals and departures," in Decision and Control (CDC),
2017 IEEE 56th Annual Conference on. IEEE, 2017, pp. 763-768.

[18] H. Huang and G. Feng, "Robust h $\infty$ synchronization of chaotic lur'e systems," Chaos: An Interdisciplinary Journal of Nonlinear Science, vol. 18 , no. 3, p. $033113,2008$.

[19] L. D. Col, S. Tarbouriech, and L. Zaccarian, "H $\infty$ control design for synchronisation of identical linear multi-agent systems," International Journal of Control, vol. 91, no. 10, pp. 2214-2229, 2018.

[20] S. Z. Khong, E. Lovisari, A. Rantzer et al., "A unifying framework for robust synchronization of heterogeneous networks via integral quadratic constraints." IEEE Trans. Automat. Contr., vol. 61, no. 5, pp. 1297-1309, 2016.

[21] F. Ceragioli, C. De Persis, and P. Frasca, "Discontinuities and hysteresis in quantized average consensus," Automatica, vol. 47, no. 9, pp. 19161928, 2011.

[22] A. Alessandri and L. Zaccarian, "Stubborn state observers for linear time-invariant systems," Automatica, vol. 88, pp. 1-9, 2018

[23] M. Cocetti, S. Tarbouriech, and L. Zaccarian, "On dead-zone observers for linear plants," 2018.

[24] D. Astolfi, A. Alessandri, and L. Zaccarian, "Stubborn iss redesign for nonlinear high-gain observers," IFAC-PapersOnLine, vol. 50, no. 1, pp. 15 422-15 427, 2017.

[25] M. Cocetti, S. Tarbouriech, and L. Zaccarian, "High-gain dead-zone observers for linear and nonlinear plants," IEEE Control Systems Letters, vol. 3, no. 2, pp. 356-361, 2019.

[26] G. Casadei, D. Astolfi, A. Alessandri, and L. Zaccarian, "Synchronization of interconnected linear systems via dynamic saturation redesign," submitted to NOLCOS, 2019, http://www.diptem.unige.it/alessandri/ submission_nolcos2019.pdf.

[27] E. D. Sontag and Y. Wang, "On characterizations of the input-to-state stability property," Systems \& Control Letters, vol. 24, no. 5, pp. 351359, 1995.

[28] C. Godsil and G. Royle, Algebraic graph theory. Springer, 2001.

[29] A. Isidori, Nonlinear Control Systems II. Communication and Control Engineering. New York, New York: Springer Verlag, 1999.

[30] R. Goebel, R. G. Sanfelice, and A. R. Teel, "Hybrid dynamical systems: modeling stability, and robustness," 2012. 